\title{
Comet-shaped sources at the Galactic center
}

\section{Evidence of a wind from the central $0.2 \mathrm{pc}$}

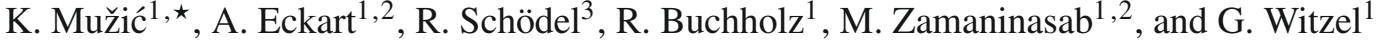 \\ 1 Physikalisches Institut, Universität zu Köln, Zülpicher Str. 77, 50937 Köln, Germany \\ e-mail:muzic@astro.utoronto.ca \\ 2 Max-Planck-Institut für Radioastronomie, Auf dem Hügel 69, 53121 Bonn, Germany \\ 3 Instituto de Astrofísica de Andalucía CSIC, Glorieta de la Astronoma S/N, 18008 Granada, Spain
}

Received 7 August 2009 / Accepted 31 May 2010

ABSTRACT

\begin{abstract}
Context. In 2007 we reported two comet-shaped sources in the vicinity of Sgr A* (0.8" and 3.4" projected distance), named X7 and X3. The symmetry axes of the two sources are aligned to within $5^{\circ}$ in the plane of the sky, and the tips of their bow shocks point towards $\mathrm{Sgr} \mathrm{A}^{*}$. Our measurements show that the proper motion vectors of both features are pointing in directions more than $45^{\circ}$ away from the line that connects them with $\mathrm{Sgr} \mathrm{A}^{*}$. This misalignment of the bow-shock symmetry axes and their proper motion vectors, combined with the high proper motion velocities of several $100 \mathrm{~km} \mathrm{~s}^{-1}$, suggest that the bow shocks must be produced by an interaction with some external fast wind, possibly coming from $\operatorname{Sgr}^{*}$, or from stars in its vicinity.

Aims. We have developed a bow-shock model to fit the observed morphology and constrain the source of the external wind.

Methods. The result of our modeling gives the best solution for bow-shock standoff distances for the two features, which allows us to estimate the velocity of the external wind, making certain that all likely stellar types of the bow-shock stars are considered.

Results. We show that neither of the two bow shocks (one of which is clearly associated with a stellar source) can be produced by the influence of a stellar wind of a single mass-losing star in the central parsec. Instead, an outflow carrying a momentum comparable to the one contributed by the ensemble of the massive young stars can drive shock velocities capable of producing the observed comet-shaped features. We argue that a collimated outflow arising perpendicular to the plane of the clockwise rotating stars (CWS) can easily account for the two features and the mini-cavity. However, the collective wind from the CWS has a scale of $>10^{\prime \prime}$. The presence of a strong, mass-loaded outbound wind at projected distances from Sgr A* of $<1^{\prime \prime}$ in fact agrees with models that predict a highly inefficient accretion onto the central black hole owing to a strongly radius dependent accretion flow.
\end{abstract}

Key words. Galaxy: center - stars: mass-loss - infrared: stars - infrared: ISM

\section{Introduction}

Analyses of stellar orbits in the central arcsecond of the Milky Way have provided indisputable evidence that the central object at the position of the radio source Sagittarius A* $\left(\mathrm{Sgr} \mathrm{A}^{*}\right)$ is a supermassive black hole (SMBH, e.g. Eckart et al. 2002; Schödel et al. 2003; Eisenhauer et al. 2005; Ghez et al. 2005, 2008; Gillessen et al. 2009). Sgr A* is located at the distance of $\sim 8 \mathrm{kpc}$, with a mass of $\sim 4 \times 10^{6} M_{\odot}$. There is long-standing evidence of outflow(s) at the Galactic center (GC). The central parsec of the Milky Way harbors a cluster of massive stars (e.g. Paumard et al. 2006) that contributes $\sim 3 \times 10^{-3} M_{\odot} \mathrm{yr}^{-1}$ to the center in the form of stellar winds (Najarro et al. 1997). However, less than $1 \%$ of this material is actually accreted onto the SMBH (Baganoff et al. 2003; Bower et al. 2003; Marrone et al. 2006) and thus has to be expelled out of the center.

The streamers of gas and dust in the central few parsecs of the Galaxy show a bubble-like region of lower density (called mini-cavity), located $\sim 3.5^{\prime \prime}$ southwest of Sgr A*. It was first pointed out on cm-radio maps by Yusef-Zadeh et al. (1990). The strong Fe[III] line emission seen toward that region (Eckart et al. 1992; Lutz et al. 1993) is consistent with gas excited

* Present address: Department of Astronomy and Astrophysics, University of Toronto, 50 St. George Street, Toronto ON M5S 3H4, Canada. by a collision with a fast $\left(\geq 1000 \mathrm{~km} \mathrm{~s}^{-1}\right)$ wind from a source within the central few arcseconds (Yusef-Zadeh \& Wardle 1993; Yusef-Zadeh \& Melia 1992).

In Mužić et al. (2007) we presented NACO/VLT multi-epoch observations at $3.8 \mu \mathrm{m}$ ( $L^{\prime}$-band) which allowed us to derive proper motions of narrow filamentary features associated with the gas and dust streamers of the mini-spiral. Proper motions of several bow-shock sources have also been reported. The analysis has shown that the shape and the motion of the filaments is inconsistent with a purely Keplerian motion of the gas in the potential of Sgr A* and that additional mechanisms must be responsible for their formation and motion. We argued that the properties of the filaments are probably related to an outflow from the disk of young mass-losing stars around Sgr A*. In part, the outflow may originate in the immediate vicinity of the black hole itself.

In Mužić et al. (2007), we reported the existence of the two comet-shaped features X3 and X7, located at projected distances of $3.4^{\prime \prime}$ and $0.8^{\prime \prime}$ from $\mathrm{Sgr} \mathrm{A}^{*}$, respectively. The symmetry axes of the two bow shocks are almost aligned (within $5^{\circ}$ ) and point towards Sgr A* (see Fig. 1). At the same time, their proper motion vectors are not pointing in the direction of the symmetry axes, as would be expected if the bow-shock shape were produced by a supersonic motion of a mass-losing star through a static interstellar medium. In general, bow-shock appearance can also be produced by an external supersonic wind interacting with 


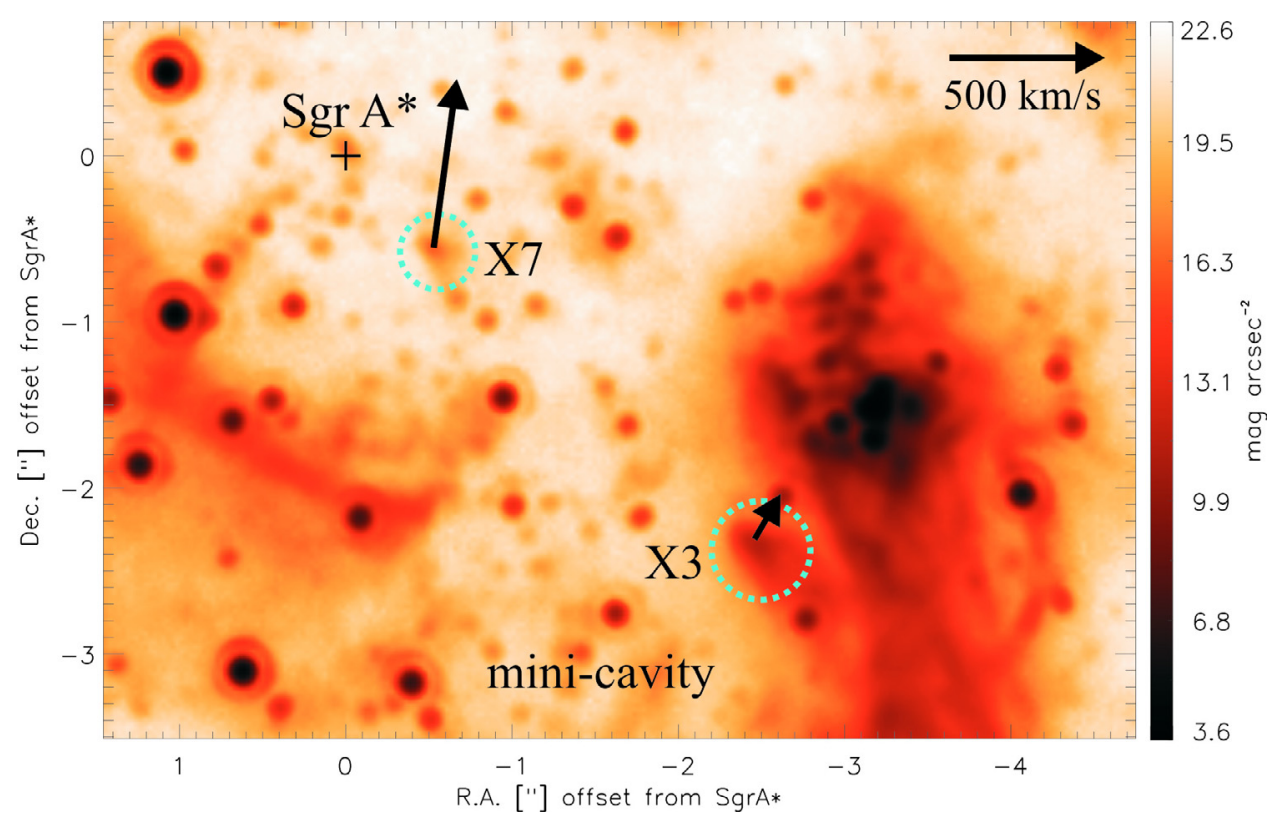

Fig. 1. NACO $L^{\prime}$-band $(3.8 \mu \mathrm{m})$ image of the Galactic center region showing projected positions of Sgr A* and the comet-shaped features X3 and $\mathrm{X} 7$. The arrows show proper motions. North is up, east is to the left.

a slower wind from a mass-losing star. If the velocity contrast of the two winds is high, one will observe a bow shock pointing towards the source of the external wind. If in addition the star moves in a different direction than the position of the external wind, the bow shock will point in the direction of the relative velocity between the star and the incident medium.

In this paper we present new proper motion measurements for the two comet-shaped sources X3 and X7 (Sect. 3). In Sect. 4 we explain details of the bow-shock modeling that was used to fit those features (Sect. 5). Possible thee-dimensional positions of the sources are discussed in Sect. 6. In Sect. 7 we argue about the nature of the two sources and the external wind held responsible for the observed arrangement. Summary and conclusions are given in Sect. 8.

\section{Observations and data reduction}

The $L^{\prime}(3.8 \mu \mathrm{m})$ images were taken with the NAOS/CONICA adaptive optics-assisted imager/spectrometer (Lenzen et al. 1998; Rousset et al. 1998; Brandner et al. 2002) at the UT4 (Yepun) at the ESO VLT. The data set includes images from 7 epochs (2002.66, 2003.36, 2004.32, 2005.36, 2006.41, 2007.25 and 2008.40) with a resolution of $\sim 100$ mas and a pixel scale of 27 mas/pixel. Data reduction (bad pixel correction, sky subtraction, flat field correction) and formation of final mosaics was performed using the DPUSER software for astronomical image analysis (T. Ott; see also Eckart \& Duhoux 1990).

\section{Proper motions}

To obtain proper motions of extended features we used the same method as described in detail in Mužić et al. (2007). Our measurements are shown in Fig. 2. The distance to the GC was assumed to be $8 \mathrm{kpc}$. The results obtained from the $L^{\prime}$-band data between 2002 and 2008, are as follows. X7: $v_{\alpha}=(-52 \pm$ 12) $\mathrm{km} \mathrm{s}^{-1}, v_{\delta}=(546 \pm 15) \mathrm{km} \mathrm{s}^{-1} . \mathrm{X} 3: v_{\alpha}=(-95 \pm 13) \mathrm{km} \mathrm{s}^{-1}$, $v_{\delta}=(122 \pm 14) \mathrm{km} \mathrm{s}^{-1}$. The uncertainties are $1 \sigma$ uncertainties of the weighted linear fit to the positions vs. time. The proper motion velocity vectors of both sources are oriented in the northwest quadrant (see Fig. 1). The results agree with results given
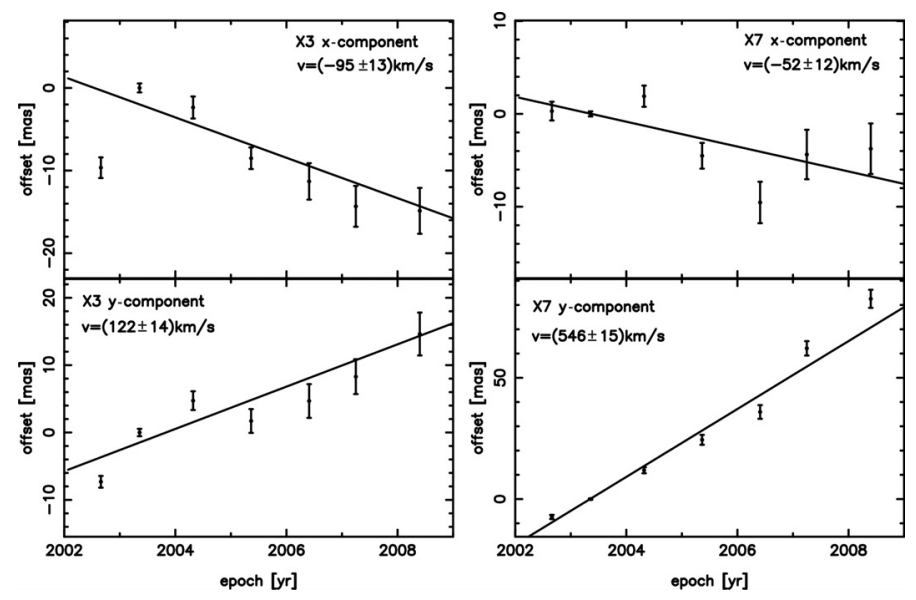

Fig. 2. $L^{\prime}$-band proper motions of the two comet-shaped features X3 and X7. The error bars show the $1 \sigma$ uncertainty of each measurement. The $x$ and $y$ components refer to RA and Dec, respectively.

in Table 1 of Mužić et al. (2007), but there is an error in their Fig. 5, where X3 is plotted as moving towards the northeast.

The $L$-band feature X7 is coincident (in projection) with a $K$-band source S 50 (Gillessen et al. 2009). The reported proper motion of $\mathrm{S} 50$ is $v_{\alpha}=(-108 \pm 5) \mathrm{km} \mathrm{s}^{-1}$ and $v_{\delta}=(361 \pm$ 7) $\mathrm{km} \mathrm{s}^{-1}$. While the orientations of the two proper motions agree, there is a significant difference in proper motion magnitudes. This, however, might give us an idea about the systematic uncertainties of the proper motions derived from the $L$-band extended sources. It seems reasonable to argue that we are not dealing with an accidental superposition of a stellar source with a dust blob along the line of sight, but that the $L$-band feature is indeed associated with the stellar source at the same position.

\section{Model}

\subsection{Analytic solution for the bow-shock shape}

Wilkin (1996) derived a fully analytic solution for the shape of a bow shock produced by a star moving through the interstellar 


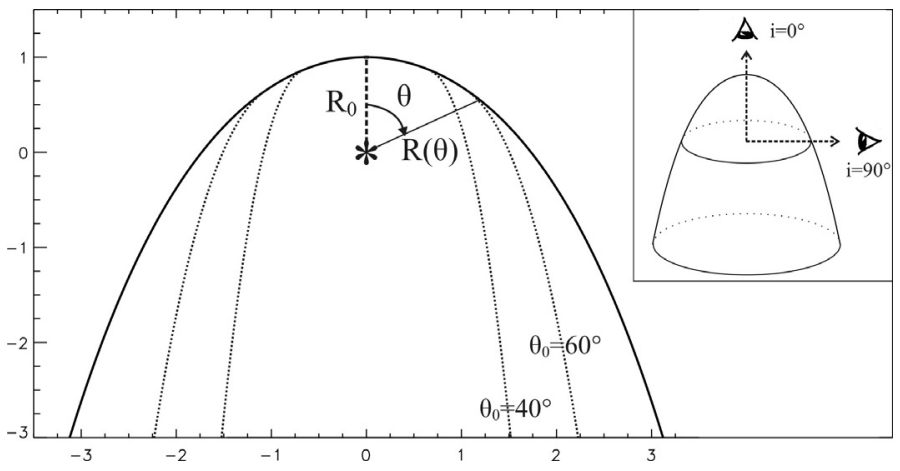

Fig. 3. The 2D bow-shock shape. Full line shows the analytic model from Wilkin (1996, see Eq. (1)). Dotted lines show narrow solutions (Zhang \& Zheng 1997), for two collimation angles $\theta_{0}=40^{\circ}$ and $\theta_{0}=60^{\circ}$. The axes are in units of $R_{0}$; the star is at the center of the coordinate system. Inset: $3 \mathrm{D}$ bow-shock sketch, explaining the conventions for the inclination angle used in this work. $i=0^{\circ}$ when the bowshock symmetry axis is parallel to the line of sight (i.e., we are looking directly at the apex); $i=90^{\circ}$ if the bow-shock symmetry axis is perpendicular to the line of sight. For a bow-shock inclined away from the observer (the observer looks at the envelope, but cannot see the apex), $90^{\circ}<i<180^{\circ}$.

medium at supersonic velocity (see Fig. 3). The 2D shell shape is given by

$R(\theta)=R_{0} \csc \theta \sqrt{3(1-\theta \cot \theta)}$,

where $\theta$ is the polar angle from the axis of symmetry, as seen by the star at the coordinate origin. Also,the so-called standoff distance obtained by balancing the ram pressures of the stellar wind and ambient medium at $\theta=0$ and is given by

$R_{0}=\sqrt{\frac{\dot{m}_{\mathrm{w}} v_{\mathrm{w}}}{4 \pi \rho_{\mathrm{a}} v_{\mathrm{a}}^{2}}}$.

Here, $\dot{m}_{\mathrm{w}}$ is the stellar mass-loss rate, $v_{\mathrm{w}}$ the terminal velocity of the stellar wind, $\rho_{\mathrm{a}}$ the ambient medium mass density, and $v_{\mathrm{a}}$ is the velocity at which the star moves through the medium (i.e. the relative velocity of the ambient medium and the star velocity in case the ambient medium is not stationary).

We assume that the shell has a thickness as shown in Fig. 3b in Mac Low et al. (1991). Then we rotate the 2D shape around its axis of symmetry to obtain the $3 \mathrm{D}$ shell.

\subsection{Narrow solution for the bow-shock shape}

The model by Wilkin (1996) incorporates the instantaneous cooling approximation: the interaction between the stellar wind and the ambient medium takes place in an infinitely thin layer, where the two flows are fully mixed and immediately cooled. In this case $R_{0}$ directly gives the distance of the star to the apex of the bow shock. However, Comerón \& Kaper (1998) point out that this might not be true if cooling of the shocked stellar wind is inefficient. In this case it is expected that the bow shock would be located at a distance somewhat greater than $R_{0}$ (Comerón \& Kaper 1998; Raga et al. 1997; Povich et al. 2008). For some GC sources, Tanner et al. (2005) indeed report that a better fit can be obtained if the apex of the bow shock is shifted away from the star. We have observed the same behavior for several other GC bow shocks.
Zhang \& Zheng (1997) have investigated the case where the wind ejection from the star is not necessarily isotropic. It is instead confined in a cone of solid angle $\Omega=2 \pi\left(1-\cos \theta_{0}\right)$, where $2 \theta_{0}$ is the opening angle in which the matter is ejected. The standoff distance is then given by

$R_{0}=\sqrt{\frac{\dot{m}_{\mathrm{w}} v_{\mathrm{w}}}{\Omega \rho_{\mathrm{a}} v_{\mathrm{a}}^{2}}}$

As can be seen in Fig. 3, with $\theta_{0}$ getting smaller, the bow shock profile will have a narrower shape. For $\theta_{0} \rightarrow 3 \pi / 4$ the shape is very similar to the one given by Eq. (1). Although this model has been developed for the case of a collimated stellar wind, we do not assume such a collimation, since it would imply that the collimation of the wind is in the direction of star's motion, relative to the ambient medium. There is no a priori reason for the stellar velocity and wind collimation direction to be linked. We introduce $\theta_{0}$ as an additional factor that allows one to control the width of the bow shock outline, while causing the apex of the bow shock to be slightly displaced away from the star.

\subsection{Generating emission maps}

To generate a simulated observation, we start by illuminating the shell by the star placed at the origin of the coordinate system and then calculate the emission along each ray intersecting the shell. The shell is inclined to the line of sight by the angle $i$, and also rotated by a position angle PA in the plane parallel to the plane of the sky. The inset in Fig. 3 explains the convention used for the inclination angle. PA is measured east of north.

Each parcel of the shell is assigned the optical depth $\tau$, calculated as

$\tau(\lambda)=\tau_{\mathrm{abs}}(\lambda)+\tau_{\mathrm{sca}}(\lambda)=L \int_{a_{-}}^{a_{+}} n_{\mathrm{d}}(a) C_{\mathrm{ext}}(a, \lambda) \mathrm{d} a$.

We assume a graphite + silicate mixture with a power-law grain size distribution $n_{\mathrm{d}}(a)$ (Mathis et al. 1977), where $a$ is the grain size. The dust extinction coefficient is $C_{\text {ext }}=\pi a^{2}\left(Q_{\mathrm{abs}}+Q_{\text {sca }}\right)$, where $Q_{\mathrm{abs}}$ and $Q_{\text {sca }}$ are dust absorption and scattering efficiencies, respectively (Laor \& Draine 1993) ${ }^{1}$. Here, $L$ is the length of the shell parcel along the line of sight.

The source X7 is polarized (see Mužić et al. 2007), suggesting that scattering of the stellar emission by dust particles in the bow-shock envelope is probably important. To account for scattering, we proceed in the following way. Emission of each parcel of the shell has contributions both from scattering and thermal emission: $L=L_{\mathrm{sca}}+L_{\mathrm{th}}$. Here, $L_{\mathrm{th}} \propto B\left(T_{\mathrm{d}}\right)\left(1-\mathrm{e}^{-\tau_{\mathrm{abs}}}\right) \epsilon_{\mathrm{th}}$ and $L_{\mathrm{sca}} \propto$ $d^{-2} \epsilon_{\text {sca }} P\left(\theta_{\text {sca }}\right) \mathrm{e}^{-\tau_{\text {sca }}}$, where $d$ is the distance from the star, $B\left(T_{d}\right)$ is the black body emission at the dust temperature, integrated over grain sizes, and over wavelengths in our observing band; $\epsilon_{\mathrm{th}}$ and $\epsilon_{\mathrm{sca}}$ are thermal emission and scattering efficiencies, respectively. Dust temperature at a distance $d$ (in parsecs) from the star can be calculated as $T_{\mathrm{d}}=27 a_{\mu \mathrm{m}}^{-1 / 6} L_{*, 38}^{1 / 6} d_{\mathrm{pc}}^{-1 / 3} \mathrm{~K}$ (Van Buren $\&$ McCray 1988; Krügel 2003), where $a_{\mu \mathrm{m}}$ is dust grain size in $\mu \mathrm{m}$, and $L_{*, 38}$ is stellar luminosity in $10^{38} \mathrm{erg} \mathrm{s} \mathrm{s}^{-1}$. The (normalized) scattering function, $P\left(\theta_{\text {sca }}\right)$, controls the amount of forward scattering, and is given by

$P\left(\theta_{\text {sca }}\right)=\frac{1-g^{2}}{1+g^{2}-2 g \cos \left(\theta_{\text {sca }}\right)}$.

1 Available at http://www . astro.princeton. edu/ draine/ 


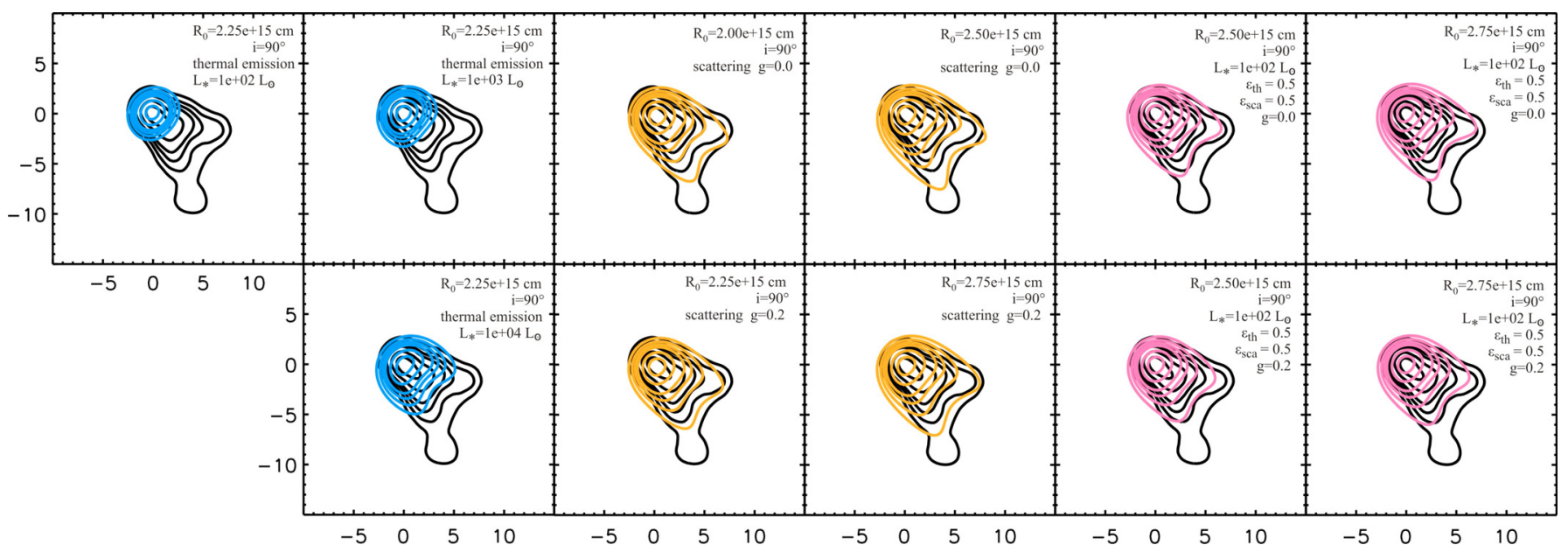

Fig. 4. Results of our modeling for the feature X7 (black contours), observed in the epoch 2003.36. The image is previously deconvolved and beam-restored to the nominal resolution of our $L^{\prime}$-band images. Color contours represent the bow-shock model projected onto the plane of the sky. $\mathrm{PA}=50^{\circ}(\mathrm{E}$ of $\mathrm{N})$, and the shape is given by the analytic solution (Sect. 4.1). We show purely thermal emission in blue (left), pure scattering in orange (middle), and equal contributions from both processes in purple (right). For each set of parameters, we show the results for two different values of $R_{0}$, to depict how change in the bow-shock size affects the fit. Contour levels are at $(20,30,40,50,70,90) \%$ of the maximum. North is up, east to the left. The axes are labeled in pixel offsets from the brightest point in the image; 1 pixel is equivalent to 0.027 mas.

For $g=0$, scattering is isotropic, and $P$ does not depend on the scattering angle $\theta_{\text {sca }} ; g=1$ means full forward scattering. We varied $g$ between 0 and 0.8 , in steps of 0.2 . Since $\epsilon_{\text {th }}$ and $\epsilon_{\text {sca }}$ are generally not known, we chose to test three different cases: $\left(\epsilon_{\mathrm{th}}, \epsilon_{\mathrm{sca}}\right)=(1.0,0.0),(0.0,1.0)$, and $(0.5,0.5)$, i.e. thermal emission only, scattering only, and equal contributions from both processes. The shape of the thermal emission depends on the dust temperature distribution, where we investigate central stars with luminosities $\left(10^{2}, 10^{3}, 10^{4}\right) L_{\odot}$.

Finally, the resulting projection of the $3 \mathrm{D}$-geometry onto the plane of the sky is rebinned to the pixel scale of the NACO images and smoothed with a Gaussian PSF having FWHM equivalent to the angular resolution of our images.

\section{Results}

\section{1. $X 7$}

Figure 4 shows the best-fit results of our bow-shock modeling for the feature $\mathrm{X} 7$. We tested the three different combinations of $\left(\epsilon_{\mathrm{th}}, \epsilon_{\mathrm{sca}}\right)$, and five values of $g=(0.0,0.2,0.4,0.6,0.8)$. In Fig. 4 , however, we show only those values that result in good fits, except for the leftmost panels, which we show to illustrate the behavior of purely thermal emission. Thermal emission is dominant in the vicinity of the star, but cannot fit the extended tail that we observe in X7, unless the central star is much brighter than the cases investigated here. This, however, is not plausible since X7 is very faint in the $K$-band (see discussion below). The tail of the bow shock is described much better by scattering. For $\left(\epsilon_{\mathrm{th}}, \epsilon_{\mathrm{sca}}\right)=(0.5,0.5)$, we only show the solution for $L_{*}=10^{2} L_{\odot}$. We choose to do so because the difference in resulting contours for the three stellar luminosities is small, and gives the same solution for the physically most interesting parameter, $R_{0}$. In cases where scattering is important $\left(\epsilon_{\mathrm{sca}} \geq 0.5\right)$, more forward scattering (large $g$ ) results in a more compact model, thus not fitting well the outer contours. There is no significant influence of the parameters on the inner contours, owing to the relatively small size of the feature and smoothing. These differences can be better observed in the case of X3.
For each set of parameters $\left(\epsilon_{\mathrm{th}}, \epsilon_{\mathrm{sca}}, g\right)$, we have tested different values of $R_{0}$. By changing $R_{0}$, the model preserves the same shape of the contours, but is as a whole expanded or shrunken. Therefore in Fig. 4 we plot two values of $R_{0}$ for each set of parameters. We chose values in the way that shows how the change in $R_{0}$ affects the fit. By changing its value by a larger amount, the fit becomes inadequate.

The best solutions for different sets of parameters are obtained for $R_{0} \approx 2.5 \times 10^{15} \mathrm{~cm}$, and $P A=50^{\circ}$, measured east of north. In this case the best results are obtained using the simple analytic 2D solution (Eq. (1)). Both X7 and X3 have an unusually narrow appearance, and therefore are best fitted with inclination angles close to $90^{\circ}$.

X7 coincides with a point source at shorter wavelengths (see discussion of proper motions in Sect. 3). Photometric measurements give $H=18.9 \pm 0.1$ and $K=16.9 \pm 0.1$ (Schödel et al. 2010). For the local extinction at the position of $X 7$ we assume $A_{K}=2.5$ (Schödel et al. 2010). In Sect. 7.1 we discuss possible stellar types and the implications this has on the external wind parameters.

\section{2. $\times 3$}

Figure 5 shows the best-fit results of our bow-shock modeling for the feature $\mathrm{X} 3$. This feature is very elongated, so a satisfactory fit cannot be obtained using the analytic $2 \mathrm{D}$ solution. It requires a narrow model (see Sect. 4.2), with small opening angles $\theta_{0}$. As in the case of X7, the outer contours are represented better in models with lower $g$, while higher $g$ values result in more compact inner contours. Here it is even more evident that thermal emission gives too compact a model. The elongated tail of X3 can only be well-fitted with models that include scattering. Therefore we only show these solutions, in pairs of two different values of $R_{0}$. The best-fit solutions give $R_{0} \approx 1.5 \times 10^{16} \mathrm{~cm}$, with $\theta_{0}=30^{\circ}, i=90^{\circ}$, and $\mathrm{PA}=55^{\circ}$.

In contrast to $\mathrm{X} 7$, there is no detectable point source at the position of $\mathrm{X} 3$ in our $K_{\mathrm{S}}$-band images. Local extinction at the position of $\mathrm{X} 3$ is $A_{K} \approx 2.7$ (Schödel et al. 2010). In Sect. 7.2 we discuss the possible nature of this source and the implications this has on the external wind parameters. 


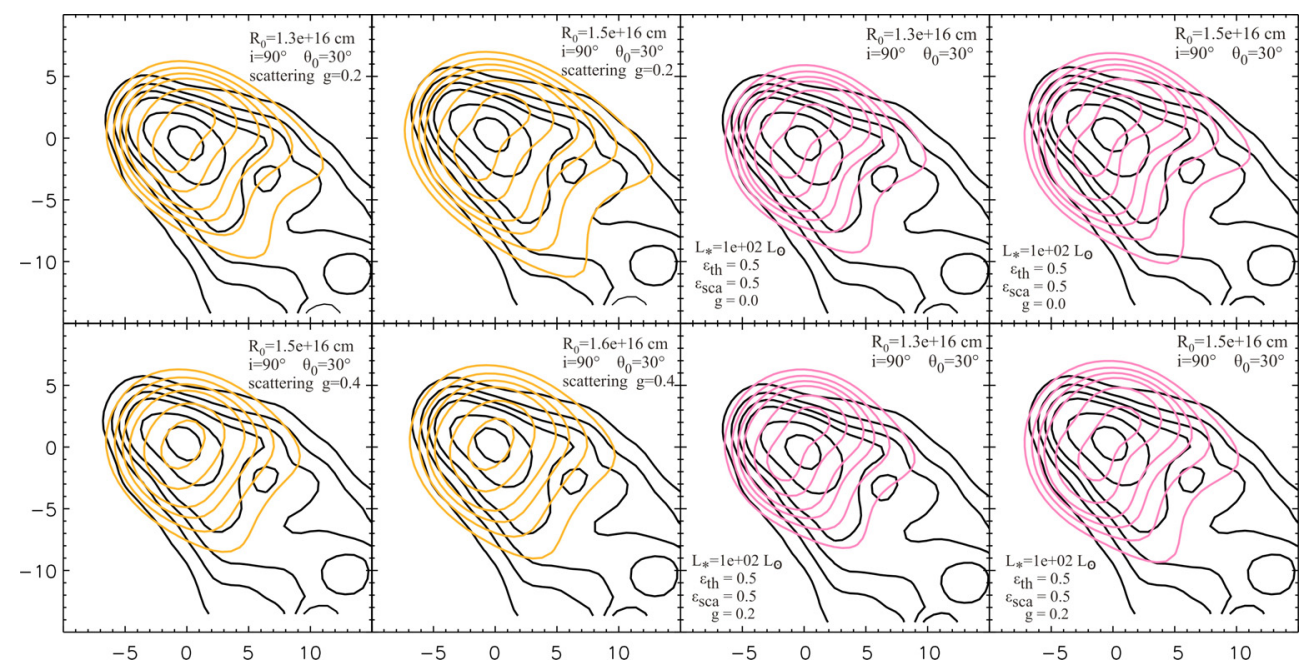

Fig. 5. Best-fit results of the modeling for the feature X3 (black contours) observed in the epoch 2003.36. The image is previously deconvolved and beam-restored to the nominal resolution of our $L^{\prime}$-band images. Color contours represent the bow-shock model projected onto the plane of the sky. $\mathrm{PA}=55^{\circ}(\mathrm{E}$ of $\mathrm{N})$ and $\theta_{0}=30^{\circ}$ in all cases. We show the pure scattering case in orange (left), and equal contributions from scattering and thermal emission in purple (right). For each set of parameters, we show the results for two different values of $R_{0}$, in order to depict how change in the bow-shock size affects the fit. Contour levels are at $(20,30,40,50,70,90) \%$ of the maximum. North is up, east to the left. The axes are labeled in pixel offsets from the brightest point in the image; 1 pixel is equivalent to 0.027 mas.

\section{The 3D arrangement}

Figure 6 shows a 3D reconstruction of some of the features found in the central parsec of the Galaxy. The shaded area represents the disk of clockwise-rotating stars (CWS, Paumard et al. 2006; Beloborodov et al. 2006; Lu et al. 2009), and the colored spheres are the stellar members. The positions of the stars and the disk parameters $\left(n_{x}, n_{y}, n_{z}\right)=(-0.12,-0.79,0.6)$ are from Paumard et al. (2006). The stars are represented by different colors according to their distance from the observer (green is closer and violet is farther away from us).

Eckart et al. (2002) show how a 3D separation $r$ from the center can be estimated by comparing the proper motion $\left(V_{\mathrm{PM}}\right)$ of a star to the 3D velocity dispersion $\sigma$. The probability that a star at the position $r$ has a proper motion greater than $V_{\mathrm{PM}}$ can be calculated via

$P\left(V>V_{\mathrm{PM}}, r\right)=1-\frac{1}{\sigma^{2}} \int_{0}^{V_{\mathrm{PM}}} v \exp \left(\frac{-v^{2}}{2 \sigma^{2}}\right) \mathrm{d} v$.

The velocity dispersion $\sigma(r)$, and hence the probability $P(V>$ $\left.V_{\mathrm{PM}}, r\right)$, decrease with increasing radii $\mathrm{r}$. For a fast-moving star it becomes increasingly unlikely that they belong to statistical samples at correspondingly larger radii. Therefore $P(V>$ $\left.V_{\mathrm{PM}}, r\right)$ can be interpreted as a measure of how likely it is that the star belongs to a sample of stars at radius $r$ or larger. Using the projected position $R$ instead of the 3D value $r$, one can calculate $P\left(V>V_{\mathrm{PM}}, R\right)$ as an upper limit of the probability $P\left(V>V_{\mathrm{PM}}, r\right)$. For a given radius $r$, we can calculate the velocity dispersion as $\sigma^{2}=G M_{\mathrm{BH}} / r$, where $M_{\mathrm{BH}}$ is the mass of Sgr A*. This gives a good estimate of $\sigma$ within the central parsec where the dynamics are dominated by the gravitational potential of Sgr A*. With this method, we estimate the line-of-sight position of X7 to be between $z=-3.2^{\prime \prime}$ and $z=3.2^{\prime \prime}$, with $P=67 \%$. The elongation of the red object in Fig. 6 reflects this range of possible $z$-positions for X7. The 3D position of the bow shock $\mathrm{X} 3$ cannot be constrained that well because of its lower proper motion and larger distance from the center. Therefore the orange object representing X3 is stretched across the entire $z$-axis.

The mini-cavity is shown in pink: in projection we approximate it with a circle of $\sim 1.2^{\prime \prime}$ radius. Paumard et al. (2004) argue that the mini-cavity is a part of the Northern Arm of the Minispiral. In their reconstruction of the streamer 3D morphology, the mini-cavity should be exactly in the plane parallel to the plane of the sky and containing Sgr A*, or be within $\sim 2.5^{\prime \prime}$ from it. This is in excellent agreement with orbital models of Zhao et al. (2009). The $\pm 2.5^{\prime \prime}$ uncertainty of the line-of-sight position of the mini-cavity is depicted as an elongation of the pink object in Fig. 6 along the z-axis.

\section{Discussion}

All the interesting physics that can result from the modeling is contained in the equation for the bow shock standoff distance $R_{0}$ (Eq. (3)). The main drawback is a lack of knowledge about the stellar types (i.e. stellar wind parameters) of the two stars. The $L^{\prime}$-band images are dominated by the thermal emission of dust. Both X3 and X7 are bright at $3.8 \mu \mathrm{m}$, but extremely faint at shorter wavelengths. Both features are apparently not embedded in the mini-spiral material, so we expect them to be intrinsically dusty.

If there was no influence by any external wind, the bow shock in our images would point in the direction of the proper motion. We understand that the $v_{\mathrm{a}}$ in Eqs. (2) and (3) is the relative velocity between the stellar velocity vector and the external wind velocity vector. Since we do not have full 3D information about the stellar velocity, but only proper motions, the values of $v_{\mathrm{a}}$ that we calculate in the following represent lower limits for the real external wind velocity. For the orientation of the projected vector $v_{\mathrm{a}}$, we assume the position angle PA of the bowshock symmetry axis (Figs. 4 and 5). By estimating $v_{\mathrm{a}}$ we can therefore determine the projected position of the external wind source responsible for the observed bow-shock morphology.

\subsection{Nature of the source at the position of $X 7$}

In the following we discuss the possible nature of the X7 star, based on its apparent brightness in the $K$-band $(16.9 \pm 0.1)$, combined with the distance modulus of 14.5 and extinction of 

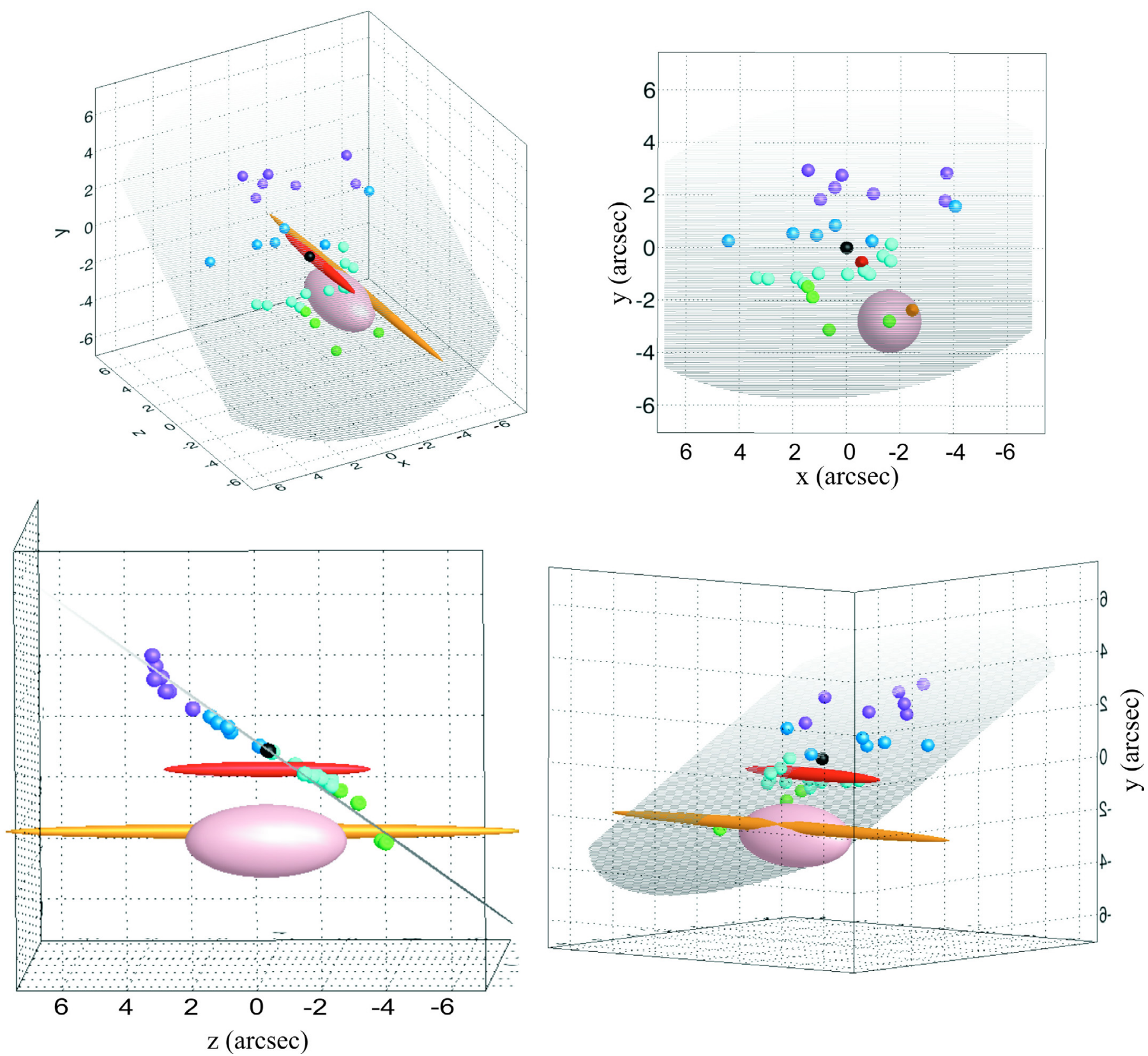

Fig. 6. Three-dimensional view of some of the Galactic center features. The axes show offsets from Sgr A* (black sphere) in arcseconds. On the $z$-axis, positive means farther away from the observer than Sgr A*. The shaded area represent the CWS disk and the colored spheres stars belonging to it. The color scheme reflects the distance from the observer, with green closest and violet farthest away from us. The bow shock sources are shown in red (X7) and orange (X3). Elongation along the $z$-axis reflects the uncertainty in the position of the two sources along the line of sight (see text). The pink spheroid represents the mini-cavity: in projection we plot it as a circle with radius $r \sim 1.2^{\prime \prime}$ and the elongation along the $z$-axis reflects the range of possible positions, as given by Paumard et al. (2004) and Zhao et al. (2009). We show the same setup from four different angles, and the top right panel shows the projection onto the plane of the sky.

$A_{K} \approx 2.5$. We discuss only stellar types that agree with the $K$-band photometry.

For the number density of the ambient medium we assume $n=26 \mathrm{~cm}^{-3}$ (Baganoff et al. 2003). This value is reasonable since $\mathrm{X} 7$ is not moving through the denser mini-spiral material.

(1) Late B-type main sequence star (B7-8V). For O and B galactic stars, the following relation holds:

$$
\log \left(\dot{m}_{\mathrm{w}} v_{\mathrm{w}} R_{\star}^{0.5}\right)=-1.37+2.07 \log \left(L_{\star} / 10^{6}\right),
$$

where $\dot{m}_{\mathrm{w}}$ is in $M_{\odot} \mathrm{yr}^{-1}, v_{\mathrm{w}}$ in $\mathrm{km} \mathrm{s}^{-1}, R_{\star}$ in $R_{\odot}$, and $L_{\star}$ in $L_{\odot}$ (Lamers \& Cassinelli 1999). For a typical B7V star with $R_{\star} \sim 4 R_{\odot}$ and $L_{\star} \sim 10^{2.5} L_{\odot}$, we then have $\dot{m}_{\mathrm{w}} v_{\mathrm{w}} \sim 10^{-9} M_{\odot} \mathrm{yr}^{-1} \mathrm{~km} \mathrm{~s}^{-1}$. This kind of weak wind would require $v_{\mathrm{a}}$ of only $15 \mathrm{~km} \mathrm{~s}^{-1}$, a negligible velocity when compared to the proper motion of X7. Also, B-type main sequence stars are dust-free objects and thus probably not good candidates for producing features like X7.

(2) Herbig Ae/Be $(\mathrm{HAe} / \mathrm{Be})$ star. This class of intermediatemass, pre-main sequence objects is characterized by strong 
wind activity and infrared excess. Line emission of $\mathrm{HAe} / \mathrm{Be}$ stars often shows prominent P-Cygni profiles, indicating powerful winds with mass-loss rates varying from $10^{-8}$ to several times $10^{-6} M_{\odot} \mathrm{yr}^{-1}$, and wind velocities of several hundred $\mathrm{km} \mathrm{s}^{-1}$ (e.g. Benedettini et al. 1998; Bouret \& Catala 1998; Nisini et al. 1995). Nisini et al. (1995) show that there is a correlation between mass-loss rate and bolometric luminosity of $\mathrm{HAe} / \mathrm{Be}$ stars. A star with $L_{\star}=10^{2.5} L_{\odot}$ would have $\dot{m}_{\mathrm{w}} \sim 10^{-7} M_{\odot} \mathrm{yr}^{-1}$. Combined with $v_{\mathrm{w}} \sim 500 \mathrm{~km} \mathrm{~s}^{-1}$, this leads to $v_{\mathrm{a}} \sim 3000 \mathrm{~km} \mathrm{~s}^{-1}$. The external wind is then blowing with the velocity $v_{\text {ext }} \sim 2800 \mathrm{~km} \mathrm{~s}^{-1}$ from the direction $\sim 60^{\circ}(\mathrm{E}$ of $\mathrm{N})$. We have to note that the existence of the pre-main sequence stars at the GC is not established. It is not yet clear how the young (few Myr old) population in the central parsec has been formed, since the tidal field of Sgr A* prevents "normal" star formation via cloud collapse (e.g. Nayakshin 2006b; Portegies Zwart et al. 2006). However, the possibility of YSO presence in the central parsec was discussed by Eckart et al. (2004) and Mužić et al. (2008).

(3) Central stars of planetary nebulae (CSPN). When all the hydrogen has been exhausted and the helium ignites in the core of a low/intermediate mass star $\left(1-8 M_{\odot}\right)$, the star reaches the asymptotic giant branch (AGB). A typical AGB star at the GC is $\sim 4$ mag brighter in the $K$-band than X7. AGB stars experience high mass-loss rates that remove most of the stellar envelope, leaving behind a stellar core. The star has now entered the post-AGB and then the planetary nebula (PN) phase. During this phase the central star remains at constant luminosity, while $T_{\text {eff }}$ rises; i.e., the star moves towards the left side of the HR diagram in a horizontal line. CSPN are characterized by small mass losses, but very fast stellar winds. A typical CSPN with $\dot{m}_{\mathrm{w}} \sim 10^{-8} M_{\odot} \mathrm{yr}^{-1}, v_{\mathrm{w}} \sim 3000 \mathrm{~km} \mathrm{~s}^{-1}$, and $R \sim R_{\odot}$ (Lamers \& Cassinelli 1999) will have $L=3 \times 10^{4} L_{\odot}$, therefore $m_{K}=17 \pm 1$ for $\log T_{\text {eff }} \sim$ (4.6-4.8). The evolution of a star from the AGB phase towards the final white dwarf stage is rapid (e.g. Blöcker 1995), and the star will remain at the required brightness for less than $1000 \mathrm{yr}$ before it becomes too faint. By adopting the typical CSPN parameters, we obtain $v_{\mathrm{a}} \sim 2300 \mathrm{~km} \mathrm{~s}^{-1}$, and $v_{\text {ext }} \sim 2100 \mathrm{~km} \mathrm{~s}^{-1}$ from the direction $\sim 62^{\circ}(\mathrm{E}$ of $\mathrm{N})$.

(4) Low-luminosity Wolf-Rayet (WR) star; [WC]-type star. Population I WR stars are massive stars in a late stage of evolution. With typical luminosities between $3 \times 10^{4}$ and $10^{6} L_{\odot}$, they are several magnitudes brighter than X7, unless the local extinction at the position of $\mathrm{X} 7$ is much higher than the estimated value of $A_{K}=2.5$. A class of CSPNe identified as WR stars share the origin with other PNe stars, but are spectroscopically classified as WR stars because emission-line spectra for both types of objects come from expanding $\mathrm{H}$-poor stellar winds. WR stars in $\mathrm{PNe}$ almost exclusively appear to be of the WC type (e.g. Gorny et al. 1995), and are labeled as [WC]. About $15 \%$ of all the observed CSPNe appear to be [WC] (Acker \& Neiner 2003). PNe stars with WR stars in their centers have, in general, larger infrared excess than normal CSPNe (Kwok 2000) and more powerful winds. Crowther (2008) reviewed the properties of [WC] stars. Typically, winds are characterized by $\dot{m}_{\mathrm{w}} \sim 10^{-7}-10^{-6} M_{\odot} \mathrm{yr}^{-1}$ and $v_{\mathrm{w}} \sim 200-2000 \mathrm{~km} \mathrm{~s}^{-1}$. Adopting the lower limit values for $\dot{m}_{\mathrm{w}}$ and $v_{\mathrm{w}}$, we obtain $v_{\mathrm{a}} \sim 1900 \mathrm{~km} \mathrm{~s}^{-1}, v_{\mathrm{ext}} \sim 1700 \mathrm{~km} \mathrm{~s}^{-1}$ from the direction $\sim 65^{\circ}(\mathrm{E}$ of $\mathrm{N})$. For the average values $\dot{m}_{\mathrm{w}} \sim 10^{-6.5}$ and $v_{\mathrm{w}} \sim$ $1000 \mathrm{~km} \mathrm{~s}^{-1}$, we have $v_{\mathrm{a}} \sim 7700 \mathrm{~km} \mathrm{~s}^{-1}, v_{\mathrm{ext}} \sim 7400 \mathrm{~km} \mathrm{~s}^{-1}$ from the direction $\sim 55^{\circ}(\mathrm{E}$ of $\mathrm{N})$.

\subsection{Nature of the source at the position of $X 3$}

In contrast to $\mathrm{X} 7$, there is no detectable point source at the position of $\mathrm{X} 3$ in our $K_{\mathrm{S}}$-band images. Crowding makes individual stars of $K_{\mathrm{S}} \approx 18$ difficult to detect within the central parsec, so we define this value as an upper limit on the brightness of X3 in the $K$-band. Here we discuss the possible nature of the star at the position of $\mathrm{X} 3$, and give possible solutions for the external wind direction and velocity.

(1) Main sequence star. If on the main sequence, a star with $K \geq$ 18 would be of type A0 or later. The mass-loss rate of lowmass stars during the core H-burning phase is very low, in the range $10^{-12}-10^{-10} M_{\odot} \mathrm{yr}^{-1}$ (Lamers \& Cassinelli 1999). In this case, any ISM streaming with the velocity on the order of $10 \mathrm{~km} \mathrm{~s}^{-1}$ could produce the bow shock with the standoff distance of X3. As in the case of X7, a main sequence star cannot be a source of a dust-rich envelope observed in the $L^{\prime}$-band, so we can rule out the main sequence nature of X3.

(2) CSPN or [WC]-type star. As a CSPN evolves on the horizontal track in the HR diagram, it maintains the same luminosity while increasing the effective temperature. The peak emission of the stellar black body shifts bluewards and the star becomes fainter in the infrared. A typical CSPN or [WC]-star discussed above for X7 would have $m_{K}>18$ for $\log T_{\text {eff }}>4$ 4.8. Adopting the typical CSPN parameters, we obtain $v_{\mathrm{a}} \sim 1520 \mathrm{~km} \mathrm{~s}^{-1}$ and $v_{\text {ext }} \sim 1540 \mathrm{~km} \mathrm{~s}^{-1}$ from the direction $\sim 61^{\circ}$ (E of N). For [WR]-star, using lower limit values as above, we obtain $v_{\mathrm{a}} \sim 1240 \mathrm{~km} \mathrm{~s}^{-1}$, and $v_{\text {ext }} \sim 1260 \mathrm{~km} \mathrm{~s}^{-1}$ from the direction $\sim 62^{\circ}$ (E of N).

(3) Dust blob. Since with the current sensitivity of our observations we do not detect a point source in the $K$-band, we cannot rule out the possibility that $\mathrm{X} 3$ is a dust blob ablated by a wind from the direction of $\mathrm{Sgr} \mathrm{A}^{*}$, rather than a stellar source. This scenario could explain the observed elongated tail. The proper motion suggests that the feature moves along with the rest of the material in the IRS13 region. In this case modeling the feature as a bow shock is superfluous.

\subsection{Nature of the external wind}

The first question that to ask is about a wind source that can drive a shock of a certain velocity over a distance of few tenths of parsec. The argument of the ram pressure of such a wind at the given distance $d$ leads to

$\rho_{\mathrm{a}} v_{\mathrm{s}}^{2}=\frac{\dot{M}_{\mathrm{w}} v_{\mathrm{w}}}{4 \pi d^{2}}$

where $\dot{M}_{\mathrm{w}}$ and $v_{\mathrm{w}}$ are the mass-loss rate and velocity of the wind. The assumption behind Eq. (8) is that the wind emanates isotropically from a point source. Let us consider the case of a single star with the isotropic wind and typical wind parameters of the GC mass-losing stars: $\dot{M}_{\mathrm{w}}=10^{-5} M_{\odot} \mathrm{yr}^{-1}$ and $v_{\mathrm{w}}=750 \mathrm{~km} \mathrm{~s}^{-1}$. This wind would be capable of driving a shock of speed $v_{\mathrm{s}}$ into a medium of number density $n_{\mathrm{H}}$ with $n_{\mathrm{H}} v_{\mathrm{s}}^{2} \approx 1.7 \times 10^{7} \times$ $\left(d\left[^{\prime \prime}\right]\right)^{-2} \mathrm{~cm}^{-3} \mathrm{~km}^{2} \mathrm{~s}^{-2}$, i.e. $v_{\mathrm{s}}=160(800) \mathrm{km} \mathrm{s}^{-1}$ at a distance $d=5^{\prime \prime}\left(1^{\prime \prime}\right)$, assuming $n_{\mathrm{H}}=26 \mathrm{~cm}^{-3}$. This is an upper limit for $v_{\mathrm{s}}$, since we have assumed that the ISM only contains atomic hydrogen. These shock speeds are comparable to the proper motion values of the two sources and it is unlikely that the resulting 
bow shock orientations would be along the same line. Therefore it is clear that a single star cannot be responsible for X3 and X7. On the other hand, a combined isotropic wind of all the masslosing stars at the GC $\left(\dot{M}_{\mathrm{w}}=10^{-3} M_{\odot} \mathrm{yr}^{-1}\right.$ and $\left.v_{\mathrm{w}}=750 \mathrm{~km} \mathrm{~s}^{-1}\right)$ would result in $n_{\mathrm{H}} v_{\mathrm{s}}^{2} \approx 1.7 \times 10^{9} \times\left(d\left[{ }^{\prime \prime}\right]\right)^{-2} \mathrm{~cm}^{-3} \mathrm{~km}^{2} \mathrm{~s}^{-2}$, i.e., $v_{\mathrm{s}}=1600(8000) \mathrm{km} \mathrm{s}^{-1}$ at a distance $d=5^{\prime \prime}\left(1^{\prime \prime}\right)$. Interestingly, these results match the above estimate of X3 and X7 being CSPNe or [WC]-stars. Also, both types of stars are very shortlived, which nicely explains the lack of other objects of the same morphology in this region.

The number of PNe expected to reside within the central parsec can be estimated as

$N_{\mathrm{PN}}=\sum_{m<8 M_{\odot}} N_{*}(m) \frac{\tau_{\mathrm{PN}}}{\tau(m)}$,

where $N_{*}(m)$ stands for the number of stars of the mass $m$ currently present in the GC population, $\tau(m)$ is a typical massdependent stellar lifetime, and $\tau_{P N}$ is the lifetime of the fast wind of the CSPNe. The latter is mass-dependent, and ranges from $<100 \mathrm{yr}$ for a $8 M_{\odot}$ star to $\sim 3 \times 10^{4} \mathrm{yr}$ for a $1 M_{\odot}$ (see e.g. Villaver et al. 2002). The summation goes over all stars with masses below $8 M_{\odot}$. We set the lower mass limit at about $1 M_{\odot}$, since the stars of lower masses have lifetimes comparable to, or longer than the Hubble time, and could not yet reach the PN phase. Figer et al. (2004) present the $K$-band luminosity function (LF) resulting from deep observations of the several HST/NICMOS fields in the GC region. To estimate the stellar counts at the faint end of the LF, we fit a power law to the number counts in the range $K \approx 16.5-19.5$, where the data are reasonably complete. We also attempt to correct for the completeness using the data from Figer et al. (1999). By fitting the LF in this range we avoid the red clump population, but also the population of massive bright stars that dominate the LF in the central parsec. For scaling, we use the observed (completeness corrected) number counts in the central parsec, at $K=17$ (Schödel et al. 2007), while accounting for the different magnitude bin sizes in Schödel et al. (2007) and Figer et al. (2004). This results in a probability of $\sim 40 \%$ to find one PNe within this region. We observe two sources in a much smaller volume, which makes it less likely that they are both of the same short-living type. This speaks in favor of X3 actually being a dust feature.

The alignment of the two features is still not explained. In the case of an isotropic wind arising from the mass-losing stars, one would expect a more random distribution of such sources around the center. Curiously, the two sources are arranged in the exact direction in which the mini-cavity is projected onto the sky. If we do not think of this arrangement as a chance configuration, this might indicate that $(i)$ all three features $(X 3, X 7$, and the mini-cavity) are produced by the same event, and (ii) there is a preferred direction in which the mass is expelled at the GC. The possibility of a collimated outflow was already discussed by Mužić et al. (2007). This outflow could also account for narrow dust filaments of the Northern Arm of the mini-spiral, as well as the $\mathrm{H}_{2}$-bright lobes of the circumnuclear disk (CND). As the authors argue, the outflow could be linked to the plane of the masslosing stars so that the matter provided by stars and not accreted onto $\mathrm{Sgr} \mathrm{A}$ * is expelled perpendicular to the plane. Having an opening angle of about $30^{\circ}$, this outflow could account for the mini-cavity, $X 3$, and $X 7$ at the same time. In this case $X 3$ and $X 7$ should be located not too far away from the plane containing Sgr A*, which is already suggested by the high inclination $\left(i \approx 90^{\circ}\right)$ of the two bow shocks to the line of sight resulting from our modeling.

\section{Summary and conclusions}

We have presented $L^{\prime}$-band observations of the two cometshaped sources in the vicinity of Sgr A*, named X3 and X7. The symmetry axes of the two sources are aligned within $5^{\circ}$ in the plane of the sky and the tips of their bow shocks point towards Sgr A*. Our measurements show that the proper motion vectors of both features are pointing in directions more than $45^{\circ}$ away from the line that connects them with Sgr A*. Proper motion velocities are high, at several $100 \mathrm{~km} \mathrm{~s}^{-1}$. This misalignment of the bow-shock symmetry axes and their proper motion vectors, together with high proper motions, suggest that the bow-shocks must be produced by an interaction with some external strong wind, possibly coming from Sgr A* or stars in its vicinity.

We developed a bow-shock model to fit the observed morphology and constrain the source of the external wind. The stellar types of the two stars are not known. Moreover, one of the features is likely not a star, but just a dust structure. It might be located at the edge of the mini-cavity, and shaped by the same wind that produces the $\mathrm{X} 7$ bow shock.

We discussed the nature of the external wind and showed that neither one of the features can arise via interaction with an external wind coming from a single, mass-losing star. Instead, the observed properties of the bow shocks provide evidence for interaction with a fast and strong wind produced probably by an ensemble of mass-losing sources. Alternatively, a possible source of the wind could be Sgr A*. Shock velocities that can result from such a combined outflow over a distance assumed for the two features $\mathrm{X} 3$ and $\mathrm{X} 7$, match the velocities required to produce the bow shocks of stars in the late evolution stages of CSPNe or [WC]-stars. Short lifetimes of such stars can explain the lack of other similar comet-shaped sources in the central parsec.

We discuss our results in the light of the partially-collimated outflow already proposed in Mužić et al. (2007) and argue that such an outflow, arising perpendicular to the CWS, can account for X3 and X7, as well as for the mini-cavity. The collective wind from the CWS has a scale of $\sim 10$ arcsec. On scales of about an arcsecond or less theoretical studies predict a radius-dependent accretion flow (e.g. Blandford \& Begelman 1999; Yuan et al. 2003). Within this region the flow of a major portion of the material originally bound for accretion onto $\operatorname{Sgr} \mathrm{A}^{*}$ is inverted and the material is expelled again towards larger radii. The presence of a strong outbound wind at projected distances from $\mathrm{Sgr} \mathrm{A}^{*}$ of only $0.8^{\prime \prime}$ (X7) with a mass load of $10^{-3} M_{\odot} \mathrm{yr}^{-1}$ does in fact agree with models that predict a highly inefficient accretion onto the central $\mathrm{BH}$ owing to a strongly radius dependent accretion flow.

Knowledge of wind parameters for two bow-shock stars is crucial for drawing more quantitative conclusions on the nature of the external outflow. Spectroscopy should therefore be the next step to confirm our hypothesis.

Acknowledgements. The authors would like to thank the referee, Dr. Mark Morris, for valuable comments and suggestions that helped to improve this work. Part of this work was supported by the Deutsche Forschungsgemeinschaft (DFG) via SFB 494. R.S. acknowledges the Ramón y Cajal program of the Spanish Ministerio de Ciencia e Innovación. M.Z. was supported for this research through a stipend from the International Max Planck Research School (IMPRS) for Astronomy and Astrophysics at the Universities of Bonn and Cologne.

\section{References}

Acker, A., \& Neiner, C. 2003, A\&A, 403, 659

Baganoff, F. K., Maeda, Y., Morris, M., et al. 2003, ApJ, 591, 891 Beloborodov, A. M., Levin, Y., Eisenhauer, F., et al. 2006, ApJ, 648, 405 Benedettini, M., Nisini, B., Giannini, T., et al. 1998, A\&A, 339, 159 
K. Mužić et al.: Comet-shaped sources at the GC

Blandford, R., \& Begelman, M. 1999, MNRAS, 303, L1

Blöcker, T. 1995, A\&A, 299, 755

Bouret, J.-C., \& Catala, C. 1998, A\&A, 340, 163

Bower, G. C., Wright, M. C. H., Falcke, H., \& Backer, D. C. 2003, ApJ, 588, 331

Brandner, W., Rousset, G., Lenzen, R., et al. 2002, Messenger, 107, 1

Comerón, F., \& Kaper, L. 1998, A\&A, 338, 273

Crowther, P. A. 2008, in Hydrogen-Deficient Stars, ed. K. Werner, \& T. Rauch, ASP Conf. Ser., 391, 83

Eckart, A., \& Duhoux, P. R. M. 1990, in Astrophysics with Infrared Arrays, ed. R. Elston (San Francisco: ASP), ASP Conf. Ser., 14, 336

Eckart, A., Genzel, R., Krabbe, A., et al. 1992, Nature, 355, 526

Eckart, A., Genzel, R., Ott, T., \& Schödel, R. 2002, MNRAS, 331, 917

Eckart, A., Moultaka, J., Viehmann, T., Straubmeier, C., \& Mouawad, N. 2004, ApJ, 602, 760

Eisenhauer, F., Genzel, R., Alexander, T., et al. 2005, ApJ, 628, 246

Figer, D. F., Sungsoo, K., Morris, M., et al. 1999, ApJ, 525, 750

Figer, D. F., Rich, M., Sungsoo, K., Morris, M., \& Serabyn, E. 2004, ApJ, 601, 319

Ghez, A. M., Salim, S., Hornstein, S. D., et al. 2005, ApJ, 620, 744

Ghez, A. M., Salim, S., Weinberg, N. N., et al. 2008, ApJ, 689, 1044

Gillessen, S., Eisenhauer, F., Trippe, S., et al. 2009, ApJ, 692, 1075

Gorny, S. K., Acker, A., Stasinska, G., Stenholm, B., \& Tylenda, R. 1995, in Proc. IAU Symp. 163, ed. K. A. van der Hucht, \& P. M. Williams, 85

Krügel, E. 2003, The Physics of Interstellar Dust (IOP Publishing), Series in Astronomy and Astrophysics

Kwok, S. 2000, The Origin and Evolution of Planetary Nebulae (Cambridge University Press)

Lamers, H. J. G. L. M., \& Cassinelli, J. P. 1999, Introduction to Stellar Winds (Cambridge University Press)

Laor, A., \& Draine, B. T. 1993, ApJ, 402, 441

Lenzen, R., Hofmann, R., Bizenberger, P., \& Tusche, A. 1998, in Infrared Astronomical Instrumentation, ed. A. M. Fowler, Proc. SPIE, 3354, 606

Lu, J. R., Ghez, A. M., Hornstein, S. D., et al. 2009, ApJ, 690, 1463
Lutz, D., Krabbe, A., \& Genzel, R. 1993, ApJ, 418, 244

Mac Low, M. M., van Buren, D., Wood, D. O. S., \& Churchwell, E. 1991, ApJ, 369,395

Marrone, D. P., Moran, J. M., Zhao, J.-H., \& Rao, R. 2006, ApJ, 640, 308

Mathis, J. S., Rumpl, W., \& Nordsieck, K. H. 1977, ApJ, 217, 425

Mužić, K., Eckart, A., Schödel, R., Meyer, L., \& Zensus, A. 2007, A\&A, 469, 993

Mužić, K., Schödel, R., Eckart, A., Meyer, L., \& Zensus, A. 2008, A\&A, 482, 173

Najarro, F., Krabbe, A., Genzel, R., et al. 1997, A\&A, 325, 700

Nayakshin, S. 2006b, in J. Phys. Conf. Ser. 54, ed. R. Schödel, G. C. Bower, M. P. Muno, S. Nayakshin, \& T. Ott, 208

Nisini, B., Milillo, A., Saraceno, P., \& Vitali, F. 1995, A\&A, 302, 169

Paumard, T., Maillard, J.-P., \& Morris, M. 2004, A\&A, 426, 81

Paumard, T., Genzel, R., Martins, F., et al. 2006, ApJ, 643, 1011

Portegies Zwart, S. F., Baumgardt, H., McMillan, S. L. W., et al. 2006, ApJ, 641, 319

Povich, M. S., Benjamin, R. A., Whitney, B. A., et al. 2008, ApJ, 689, 242

Raga, A. C., Noriega-Crespo, A., Cantó, J., et al. 1997, RMxAA, 33, 73

Rousset, G., Lacombe, F., Puget, P., et al. 1998, in Adaptive Optical System Technologies, ed. D. Bonaccini, Proc. SPIE, 3353, 516

Schödel, R., Ott, T., Genzel, R., et al. 2003, ApJ, 596, 1015

Schödel, R., Eckart, A., Alexander, T., et al. 2007, A\&A, 469, 125

Schödel, R., Najarro, F., Muzic, K., \& Eckart, A. 2010, A\&A, 511, A18

Tanner, A., Ghez, A. M., Morris, M. R., \& Christou, J. C. 2005, ApJ, 624, 742

Van Buren, D., \& McCray, R. 1988, ApJ, 329, L93

Villaver, E., Manchado, A., \& García-Segura, G. 2002, ApJ, 581, 1204

Wilkin, F. P. 1996, Models of Interacting Stellar Winds, Ph.D. Thesis, University of California, Berkley

Yuan, F., Quataert, E., \& Narayan, R. 2003, ApJ, 598, 301

Yusef-Zadeh, F., \& Melia, F. 1992, Ap, 385, L41

Yusef-Zadeh, F., \& Wardle, M. 1993, ApJ, 405, 584

Yusef-Zadeh, F., Morris, M., \& Ekers, R. D. 1990, Nature, 348, 45

Zhang, Q., \& Zheng, X. 1997, ApJ, 474, 719

Zhao, J.-H., Morris, M. R., Goss, W. M., \& An, T. 2009, ApJ, 699, 186 